\title{
The role of RhoC in epithelial-to-mesenchymal transition of ovarian carcinoma cells
}

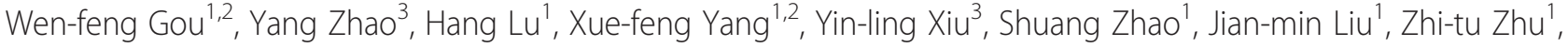
Hong-zhi Sun ${ }^{1}$, Yun-peng Liu ${ }^{4}$, Feng $X^{5}{ }^{5}$, Yasuo Takano ${ }^{6}$ and Hua-chuan Zheng ${ }^{1,2^{*}}$

\begin{abstract}
Background: RhoC is a small G protein/GTPase and involved in tumor mobility, invasion and metastasis. Previously, up-regulated RhoC expression is found to play an important role in ovarian carcinogenesis and subsequent progression by modulating proliferation, apoptosis, migration and invasion.

Methods: We transfected RhoC-expressing plasmid and RhoC siRNA into CAOV3 and OVCAR3 cells respectively. These cells and transfectants were exposed to vascular epithelial growth factor (VEGF), transforming growth factor (TGF)- $\beta 1$ or their receptor inhibitors with the phenotypes and their related-molecules examined.

Results: TGF- $\beta 1 R$ or VEGFR inhibitor suppressed the proliferation, migration, invasion and lamellipodia formation, the expression of N-cadherin, a-SMA, snail and Notch1 mRNA or protein, and enhanced E-cadherin mRNA and protein expression in CAOV3 and its RhoC-overexpressing transfectants, whereas both growth factors had the opposite effects in OVCAR3 cells and their RhoC-hypoexpressing transfectants. Ectopic RhoC expression enhanced migration, invasion, lamellipodia formation and the alteration in epithelial to mesenchymal transition (EMT) markers of CAOV3 cells regardless of the treatment of VEGFR or TGF- $\beta 1 \mathrm{R}$ inhibitor, whereas RhoC knockdown resulted in the converse in OVCAR3 cells even with the exposure to VEGF or TGF- $\beta 1$.
\end{abstract}

Conclusion: RhoC expression might be involved in EMT of ovarian epithelial carcinoma cells, stimulated by TGF- $\beta 1$ and VEGF.

Keywords: Ovarian carcinoma, RhoC, Epithelial-to-mesenchymal transition

\section{Background}

Ovarian cancer is the second leading cancer in women and the 5th leading cause of cancer-related deaths in women [1]. Ovarian cancer is disproportionately deadly because no sophisticated approach for the early diagnosis makes most ovarian cancers diagnosed at advanced stages, which determines the five-year survival rate of ovarian cancer comparatively low [2]. The existence of cancer stem-like cells from epithelial to mesenchymal transition (EMT) makes ovarian cancer more frequently recurrent and drug-resistant [3].

EMT is a process that epithelial cells are converted from a phenotypic shift from cells with tight cell-cell

\footnotetext{
* Correspondence: zheng_huachuan@hotmail.com

'Cancer Research Center, The First Affiliated Hospital of Liaoning Medical University, 121001 Jinzhou, China

${ }^{2}$ Key Laboratory of Brain and Spinal Cord Injury of Liaoning Province, The First Affiliated Hospital of Liaoning Medical University, 121001 Jinzhou, China Full list of author information is available at the end of the article
}

junctions, clear basal and apical polarity, and sheet-like growth architecture into spindle-like and motile cells, which is associated with cancer progression, cell invasion, chemotherapeutic resistance and the formation of side populations of cancer stem-like cells [4]. EMT is triggered by the interplay of extracellular signals (collagen, hyaluronic acid and integrin), such secreted factors as transforming growth factor (TGF)- $\beta$, vascular endothelial growth factor (VEGF), epithelial growth factor, hepatocyte growth factor, Wnt proteins and matrix metalloproteinases. The receptor-mediated signal pathways involve Akt, glycogen synthase kinase-3, Rho-GTPases and Smad, finally to up-regulate a set of transcription factors including Snai1, Slug, Zeb1, Zeb2, Goosecoid, and forkhead box protein $\mathrm{C} 2$, which regulate the expression of epithelial and mesenchymal markers at a transcriptional level [4-6]. Consequently, there appear down-regulation 
of epithelial markers (E-cadherin, desmoplakin and plakoglobin) and up-regulation of mesenchymal markers (N-cadherin, fibronectin and $\alpha$-SMA). E-cadherin loss might lead to the disruption of cell-cell adhesion and the translocation of $\beta$-catenin into the nucleus [4].

Reportedly, either up-regulation or increased activity of RhoC promotes the invasive potential of cancer cells, which is closely associated with EMT [7]. RhoC is a small ( $\sim 21-25 \mathrm{kDa}) \mathrm{G}$ protein/GTPase which belongs to the Rac subfamily of Rho family. It shuttles between inactive GDP-bound and active GTP-bound states and serves as a molecular switch in signal transduction cascades [8]. It has been found that $\mathrm{RhoC}$ promotes reorganization of the actin cytoskeleton, regulates cell shape and attachment, and coordinates cell motility and actomyosin contractility. RhoC overexpression is associated with cell invasion and metastasis of ovarian cancer $[9,10]$. RhoC-deficient mice can still develop tumors, which however fail to metastasize, arguing that $\mathrm{RhoC}$ is essential for metastasis [11]. In cervical carcinoma cells, both Notch1 and RhoC have similar phenotypic contribution to EMT, and Notch1 inhibition decreases RhoC activity, suggesting that RhoC functions as an effector of Notch1 [12]. Sequeira et al. [13] demonstrated that RhoC inactivation resulted in morphological changes of mesenchymal to epithelial transition and was accompanied by decreased direct migration and invasion of human prostate cancer cells. Bellovin et al. [14] reported that RhoC expression and activation are induced by EMT of colon carcinoma cell and RhoC promotes post-EMT cell migration.

Previously, we found that the RhoC mRNA and protein were significantly higher in ovarian cancer, and correlated with clinicopathological staging [9]. The RhoC knockdown resulted in a low growth, $\mathrm{G}_{1}$ arrest, apoptotic induction of OVCAR3 cells with the decreased expression of $A k t$, stat-3, bcl-xL and survivin, and the increased expression of Bax and Caspase-3 [10]. Here, we aimed to clarify the role of RhoC in EMT process of ovarian carcinoma, stimulated by TGF- $\beta 1$ and VEGF.

\section{Methods}

\section{Plasmid construction}

RhoC was amplified using the template of OVCAR3 cDNA and inserted into pBluescript-K by Hinc II. The primers of RhoC were forward: 5' - CCGGAATTCATGGCTGCAA TCCGA AA-3' and reverse: 5'-CGCGGATCCTCAGAG AATGGGACAGC-3'. Target RhoC DNA was digested and inserted into pEGFP-N1 between EcoR I and BamH I.

\section{Cell culture and transfection}

Ovarian carcinoma cell lines, CAOV3 (serous adenocarcioma), OVCAR3 (serous cystic adenocarcinoma), SKOV3 (serous papillary cystic adenocarcinoma), HO8910 (serous cystic adenocarcinoma), and ES-2 (clear cell carcinoma) have been purchased from ATCC. They were maintained in RPMI 1640 (ES-2, HO8910 and OVCAR3), DMEM (CAOV3) and McCoy's 5A (SKOV3) medium supplemented with 10\% fetal bovine serum (FBS), 100 units $/ \mathrm{mL}$ penicillin, and $100 \mu \mathrm{g} / \mathrm{mL}$ streptomycin in a humidified atmosphere of $5 \% \mathrm{CO}_{2}$ at $37^{\circ} \mathrm{C}$.

The ovarian carcinoma cells were treated with $R$ hoCexpressing plasmid by Attractene Transfection Reagent (QIAGEN) with pEGFP-N1 as a mock or RhoC siRNA (Sigma, USA) by HiPerFect Transfection Reagent (QIAGEN). The target sequences of RhoC siRNA were

Table 1 Primers' design for RT-PCR

\begin{tabular}{|c|c|c|c|c|c|}
\hline Names & Primer's sequence & Distribution & AT $\left({ }^{\circ} \mathrm{C}\right)$ & Product size(bp) & Extension time(s) \\
\hline \multirow[t]{2}{*}{$N$-cadherin } & F:5'-GAAAGACCCATCCACG- 3' & NM-031333.1 & 60 & 217 & 34 \\
\hline & R: 5'-CCTGCTCACCACCACTA- 3' & $2365-2581$ & & & \\
\hline \multirow[t]{2}{*}{ E-cadherin } & F:5'-CCGCCATCGCTTACA-3' & NM-057374.2 & 60 & 262 & 34 \\
\hline & R:5'-GGCACCTGACCCTTGTA-3' & $1017-1278$ & & & \\
\hline \multirow[t]{2}{*}{ a-SMA } & F:5'-GAGCGTGAGATTGTCCG-3' & NM-007392.2 & 60 & 232 & 34 \\
\hline & R: 5'-TGCTGTTGTAGGTGGTTTC-3' & $583-814$ & & & \\
\hline \multirow[t]{2}{*}{ Snail } & F:5'-GGCTCAGTTCGTAAAGG-3' & NM-001032543.1 & 60 & 357 & 34 \\
\hline & R:5'-GCAGCGGTAGTCCACA-3' & $7-363$ & & & \\
\hline \multirow[t]{2}{*}{ Slug } & F:5'-ATGCCTGTCATACCACAA-3' & FBgn0028564 & 60 & 173 & 34 \\
\hline & R: 5'-GAGGAGGTGTCAGATGGA-3' & $290-462$ & & & \\
\hline \multirow[t]{2}{*}{ RhoC } & F:5'-TGCCTCCTCATCGTCTTCA-3' & NM-001042678.1 & 60 & 310 & 34 \\
\hline & R: 5'-GCCTCAGGTCCTTCTTATTCC-3' & $391-700$ & & & \\
\hline \multirow[t]{2}{*}{ GAPDH } & F: 5'-CAATGACCCCTTCATTGACC-3' & NM_ 002046.3 & 60 & 135 & 34 \\
\hline & R: 5'- TGGAAGATGGTGATGGGATT-3' & $201-335$ & & & \\
\hline
\end{tabular}


Table 2 Antibodies' used in Western blot

\begin{tabular}{llllll}
\hline Names & Species & MW & Dilution & Code & Source \\
\hline E-Cadherin & Rabbit & $97 \mathrm{kDa}$ & $1: 1000$ & ab53033 & abcam, USA \\
N-Cadherin & mouse & $100 \mathrm{kDa}$ & $1: 1000$ & ab98952 & abcam, USA \\
a-SMA & mouse & $42 \mathrm{kDa}$ & $1: 1000$ & ab3280 & abcam, USA \\
Slug & rabbit & $30 \mathrm{kDa}$ & $1: 1000$ & ab27568 & abcam, USA \\
Notch1 & goat & $300 \mathrm{KD}$ & $1: 500$ & sc-6014 & Santa cruz, USA \\
RhoC & goat & $24 \mathrm{KD}$ & $1: 500$ & sc-26481 & Santa cruz, USA \\
B-actin & mouse & $42 \mathrm{kDa}$ & $1: 2000$ & sc-47778 & Santa cruz, USA \\
\hline
\end{tabular}

5'-GUGCCUUUGGCUACCUUGAdTdT-3' (sense) and 5'-UCAAGGUAGCCAAAGGCA CdTdT-3' (anti-sense). The negative siRNA control sequences were $5^{\prime}$-UUCU CCGAACGU GUCACGUT T-3' (sense) and 5' -ACGUG ACACGUUCGGAGAATT-3' (anti-sense). Cells were treated by recombinant human TGF- $\beta 1$ and VEGF165 (Perotech), VEGF receptor inhibitor BIBF1120 and TGF- $\beta 1$ receptor inhibitor SB431542 (Selleckchem). All cells were harvested by centrifugation, rinsed with phosphate buffered saline (PBS), and subjected to RNA and protein extraction.
A

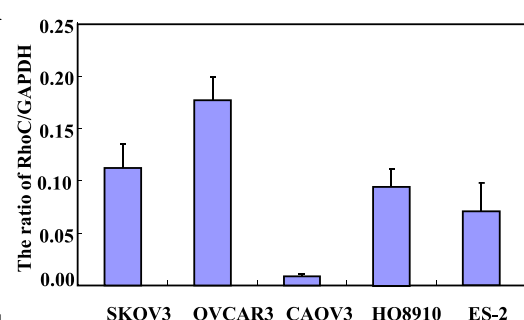

C

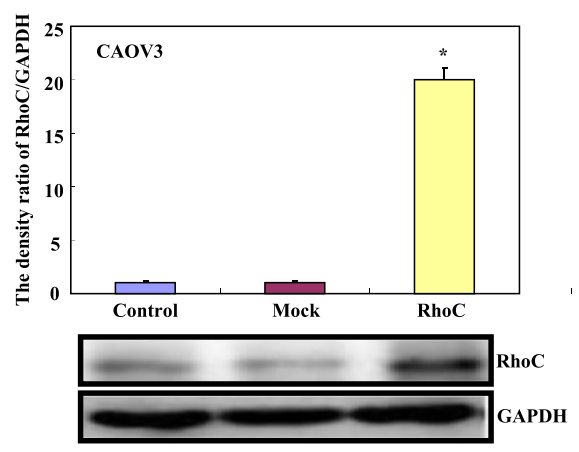

$\mathbf{E}$
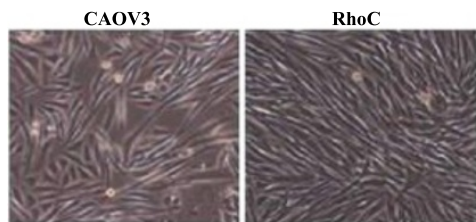

F

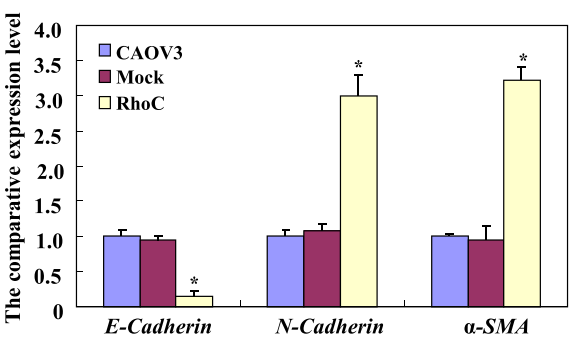

B

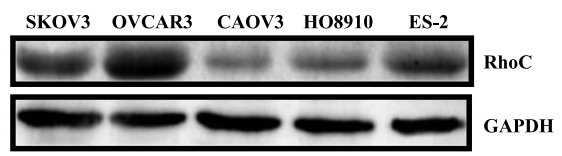

D

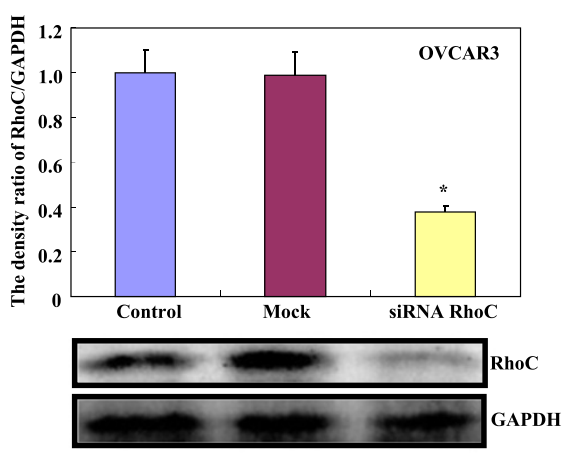

OVCAR3 RhoC siRNA
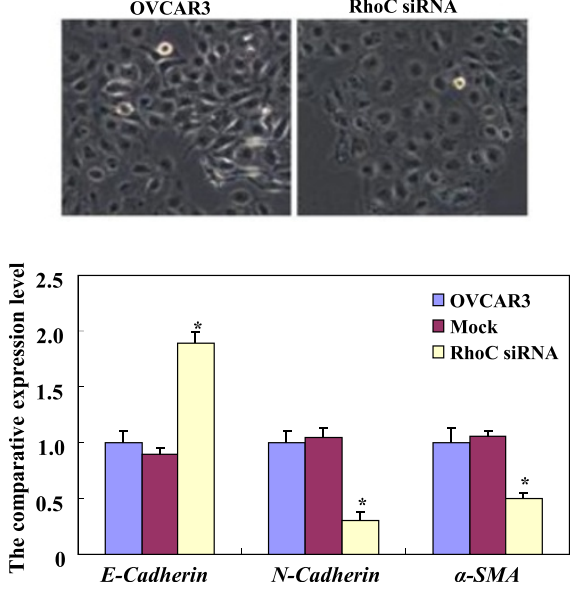

Figure 1 The involvement of RhoC in EMT of ovarian carcinoma cells. The mRNA and protein expression of RhoC was screened in ovarian carcinoma cells (SKOV3, OVCAR3, CAOV3, HO8910 and ES-2) by real-time PCR (A) and Western blot (B). CAOV3 cells were transfected with RhoC-expressing plasmid and confirmed by real-time PCR and Western blot (C). After transfection of RhoC siRNA, RhoC expression became weaker in OVCAR3 by real-time PCR and Western blot (D). CAOV3 cells became spindle after ectopic RhoC expression, while RhoC knockdown caused OVCAR3 morphorlogically round (E). There was a down-regulated expression of E-cadherin mRNA, and up-regulated expression of $N$-cadherin and a-SMA mRNA in CAOV3 transfectants by real-time PCR (F). After the treatment of RhoC siRNA, there was an increased expression of E-cadherin mRNA in OVCAR3 cells by real-time PCR, while the converse was true for the expression of N-cadherin and a-SMA mRNA (F). * compared with control and mock, $\mathrm{p}<0.05$. 


\section{Proliferation assay}

Cell counting Kit-8 (CCK-8, Japan) was employed to determine the number of viable cells. In brief, $2.5 \times$ $10^{3}$ cells/well were seeded on 96-well plate and allowed to adhere. At different time points, $10 \mu \mathrm{L}$ of CCK-8 solution was added into each well of the plate and the plates were incubated for $3 \mathrm{~h}$ and measured at $450 \mathrm{~nm}$.

\section{Wound healing assay}

Cells were seeded at a density of $1.0 \times 10^{6}$ cells/well in 6 -well culture plates. After they had grown at the confluence of $70-80 \%$, the cell monolayer in each well was scraped with a pipette tip to create a scratch, washed by PBS for three times and cultured in the FBS-free medium. Cells were photographed at $48 \mathrm{~h}$ and the scratch area was measured using Image software.
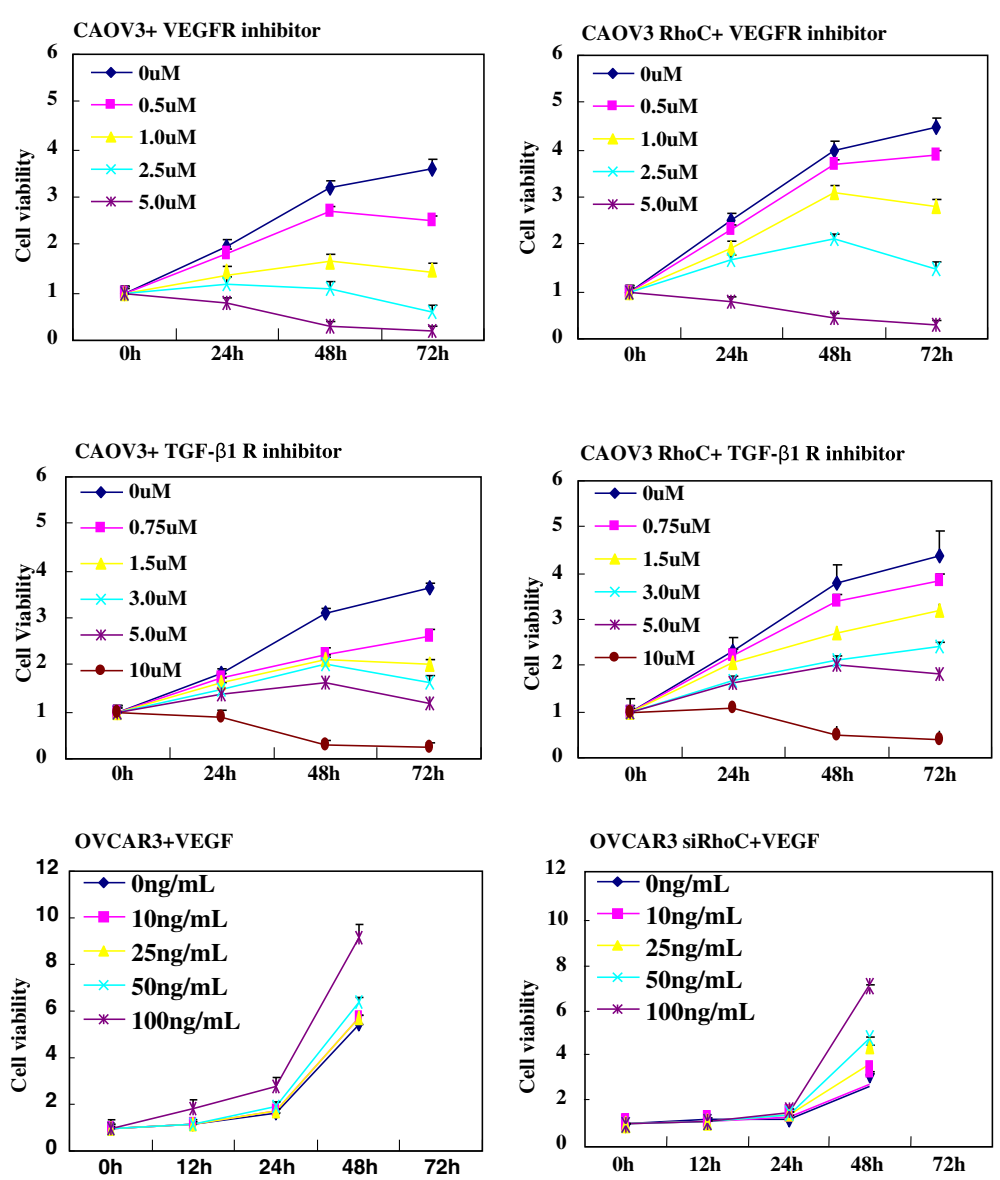

OVCAR3+TGF- $\beta 1$
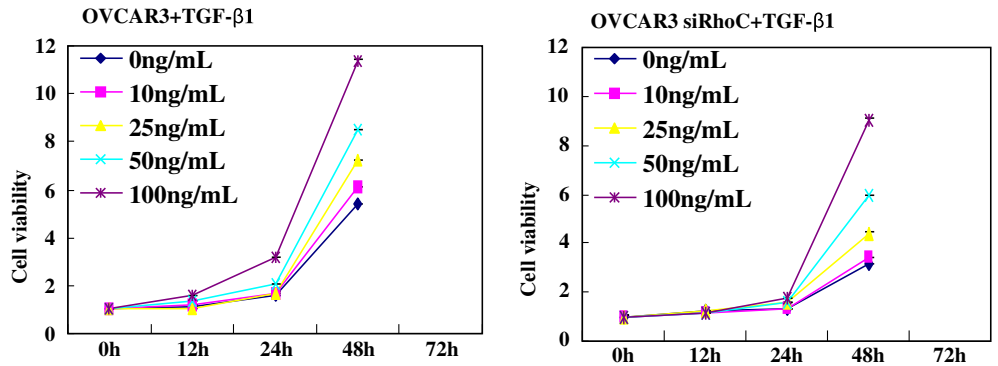

Figure 2 The RhoC-mediated effects of TGF- $\beta 1$ and VEGF on proliferation of ovarian carcinoma cells. VEGFR or TGF- $\beta 1 R$ inhibitor could suppress the proliferation of CAOV3 in both dose-dependent and time-dependent manners, but both factors promoted the proliferation of OVCAR3. VEGFR inhibitor $(2.5 \mu \mathrm{M})$, TGF- $\beta 1 R$ inhibitor $(5.0 \mu \mathrm{M})$, VEGF $(100 \mathrm{ng} / \mathrm{mL})$ and TGF $\beta 1$ $(100 \mathrm{ng} / \mathrm{mL})$ were employed to treat these ovarian carcinoma cells in the following experiments of Figures $3,4,5$, and 6. 


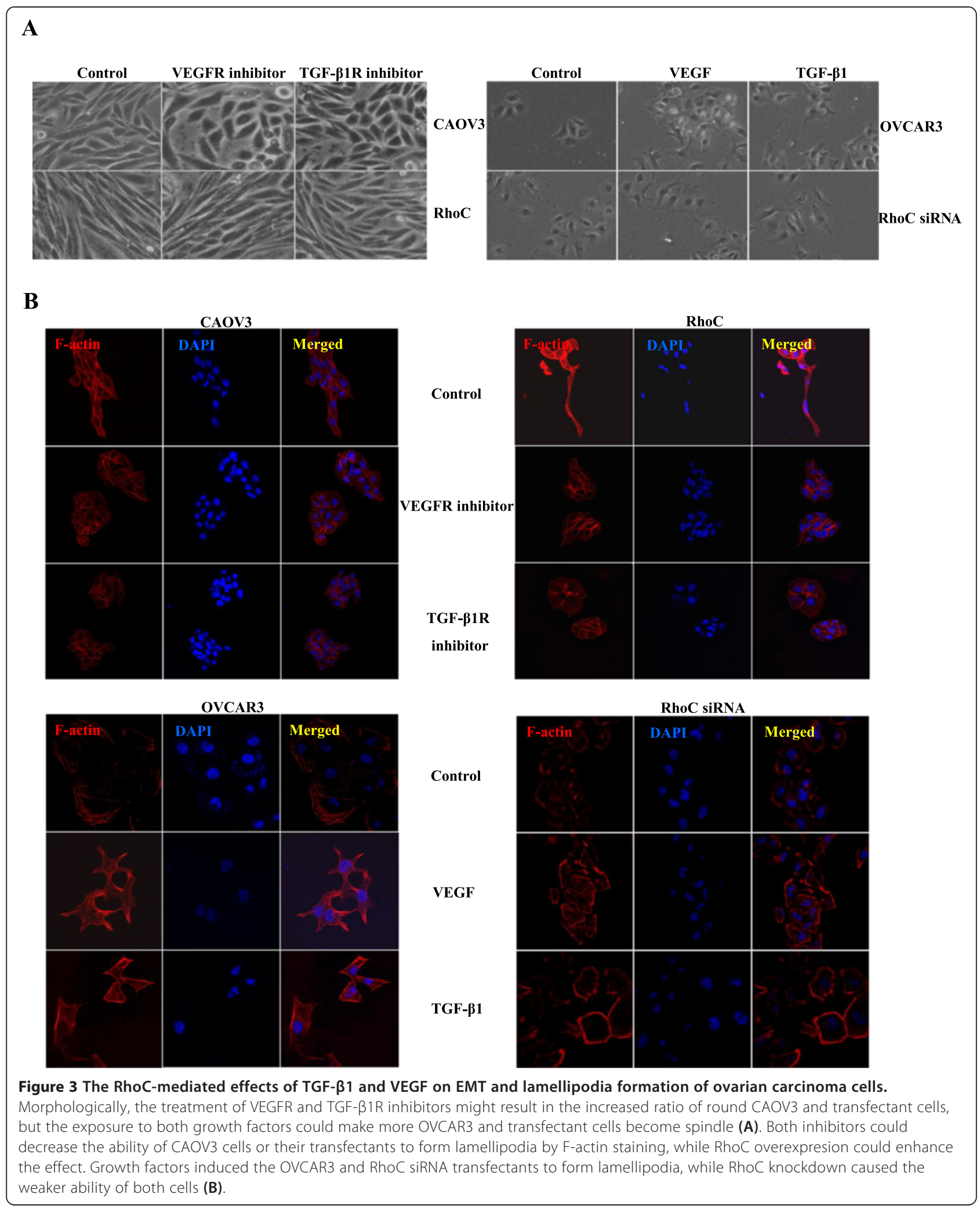

\section{Cell invasion assays}

For invasive assay, $2.5 \times 10^{5}$ cells were resuspended in serum-free DMEM or RPMI 1640 medium, and seeded in the matrigel-coated insert on the top portion of the chamber (Corning). The lower compartment of the chamber contained 10\% FBS as a chemoattractant. 


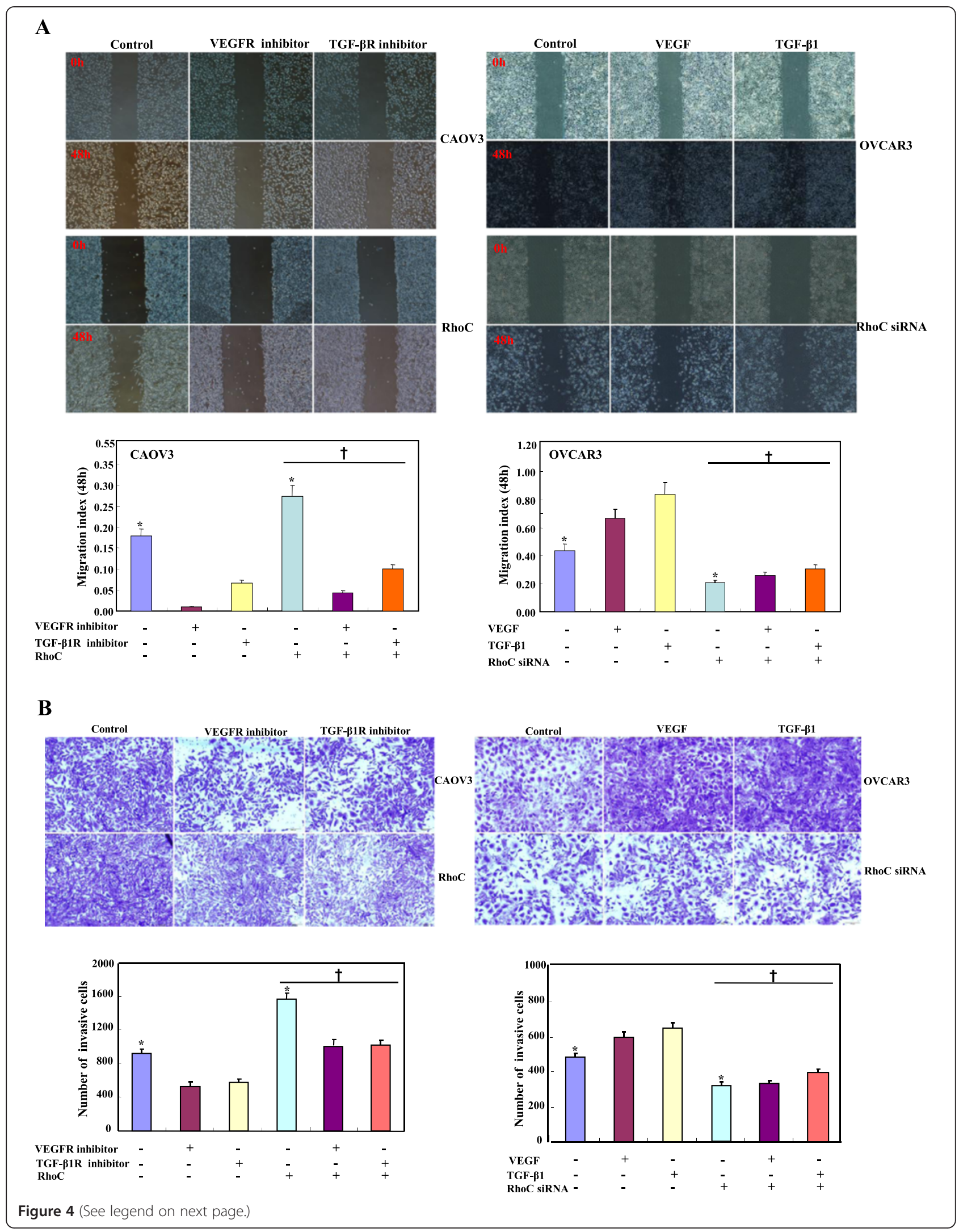


(See figure on previous page.)

Figure 4 The RhoC-mediated effects of TGF- $\beta 1$ and VEGF on migration and invasion of ovarian carcinoma cells. Inhibitors could decrease the ability of CAOV3 cells or their transfectants to migrate by wound healing assay (A) and invade by transwell (B), while RhoC overexpresion could enhance the effects. Growth factors caused the OVCAR3 and RhoC siRNA transfectants to highly migrate (A) and invade (B), while RhoC knockdown caused the weaker abilities of both cells (A and $\mathbf{B}) .{ }^{*}$ compared with treating groups, $\mathrm{p}<0.05$. + compared with corresponding either RhoC- overexpressing or -hypoexpressing group.

After incubated at $37^{\circ} \mathrm{C}$ and $5 \% \mathrm{CO}_{2}$ for $24 \mathrm{~h}$, filter inserts were removed from the wells. Cells on the upper surface of the filter were removed using a cotton swab. Those on the lower surface were fixed with $20 \%$ methanol in PBS, stained with Giemsa dye for the measurement.

\section{Immunofluorescence}

Cells were grown on glass coverslips and treated as described in the figure legends. Cells were washed twice with PBS, fixed with $4 \%$ formaldehyde for $10 \mathrm{~min}$, and permeabilized with $0.2 \%$ Triton X-100 for $10 \mathrm{~min}$. After washing with PBS, cells were incubated overnight at $4^{\circ} \mathrm{C}$ with the rabbit antibody against E-cadherin (Abcam) and the mouse antibody against N-cadherin (Abcam). They were then washed with PBS, and incubated with anti-mouse Alexa Fluor 594 (red) IgG and anti-rabbit Alexa Fluor 488 (green) IgG (Invitrogen). Alexa Fluor ${ }^{\circ}$ 594 phalloidin (red, invitrogen) for F-actin staining was employed to observe the lamellipodia. Nuclei were stained with $1 \mu \mathrm{g} / \mathrm{mL}$ DAPI (Sigma) for $30 \mathrm{~min}$ at $37^{\circ} \mathrm{C}$. Finally, coverslips were mounted with SlowFade ${ }^{\circ}$ Gold antifade reagent (invitrogen) and observed under laser confocal scanning microscope (Leica). Densitometric quantification of protein immunoreactivity was performed using Image-pro plus software (Media Cybernetics, Netherlands).

\section{Real-time RT-PCR}

Total RNA was extracted from ovarian carcinoma cell lines using Trizol (Takara, Japan) according to the manufacturer's protocol. Two micrograms of total RNA was subjected to cDNA synthesis using AMV reverse transcriptase and random primer (Takara, Japan). According to Genbank, oligonucleotide primers for PCR were designed and shown in Table 1. Real-time PCR amplification of cDNA was performed in $20 \mu \mathrm{L}$ mixtures according to the protocol of SYBR Premix Dimer Eraser kit (Takara) with GAPDH as an internal control. The expression level was expressed as $2^{-\Delta \mathrm{Ct}}$, where $\Delta \mathrm{Ct}=\mathrm{Ct}$ (gene) - Ct (GAPDH). Additionally, the expression level of the control cells was considered as " 1 ".

\section{Western blot}

Total protein was extracted by sonication in radioimmunoprecipitation assay(RIPA) buffer $(50 \mathrm{mM}$ Tris- $\mathrm{HCl}$
pH 7.5, $150 \mathrm{mM} \mathrm{NaCl}, 5 \mathrm{mM}$ EDTA, 0.5\% Nonidet P-40, $5 \mathrm{mM}$ dithiothreitol, $10 \mathrm{mM} \mathrm{NaF}$, protease inhibitor cocktail). One hundred or seventy $\mu \mathrm{g}$ denatured protein was separated on an SDS-polyacrylamide gel and transferred to Hybond membrane (Amersham, Germany), which was then blocked overnight in 5\% skim milk in tris buffered saline with Tween 20 (TTBS, $10 \mathrm{mM}$ Tris- $\mathrm{HCl}, 150 \mathrm{mM}$ $\mathrm{NaCl}, 0.1 \%$ Tween 20). For immunobloting, the membrane was incubated for 15 min with the primary antibody (Table 2). Then, it was rinsed by TBST and incubated with anti-mouse, anti-rabbit or anti-goat IgG conjugated to horseradish peroxidase (DAKO, USA, 1:1000) for $15 \mathrm{~min}$. All the incubations were performed in a microwave oven to allow intermittent irradiation [15]. Bands were visualized with LAS4010 (GE healthcare Life Science, USA) by ECL-Plus detection reagents (Santa Cruz, USA). After that, membrane was washed with WB Stripping Solution ( $\mathrm{pH} 2-3$, Nacalai, Tokyo, Japan) for $1 \mathrm{~h}$ and treated as described above except mouse anti-GAPDH antibody (Sigma, 1:10,000). Densitometric quantification of protein bands was performed with GAPDH as an internal control using Image J (NIH, USA).

\section{Statistical analysis}

All the experiments were repeated for three times and all data were showed as a mean \pm standard deviation. Statistical evaluation was performed using Mann-Whitney $\mathrm{U}$ to differentiate the means of different groups. $P<0.05$ was considered as statistically significant. SPSS 10.0 software was employed to analyze all data.

\section{Results}

The role of RhoC in EMT of ovarian carcinoma cells

As shown in Figure $1 \mathrm{~A}$ and $\mathrm{B}$, RhoC was strongly expressed in SKOV3, OVCAR3, HO8910, and ES-2, but weakly expressed in CAOV3 at both the mRNA and protein levels. Therefore, we selected CAOV3 for RhoCexpressing plasmid transfection and OVCAR3 for RhoC siRNA treatment. In comparison with the control and mock, RhoC overexpression was detected in CAOV3 cells after plasmid transfection at both the mRNA and protein levels (Figure $1 \mathrm{C}, \mathrm{p}<0.05$ ). After siRNA treatment, RhoC expression became weaker in OVCAR3 transfectants than control and mock cells by real-time PCR and Western blot (Figure 1D, $\mathrm{p}<0.05$ ). Compared with the control and mock, siRNA transfectants had a round appearance under 
A
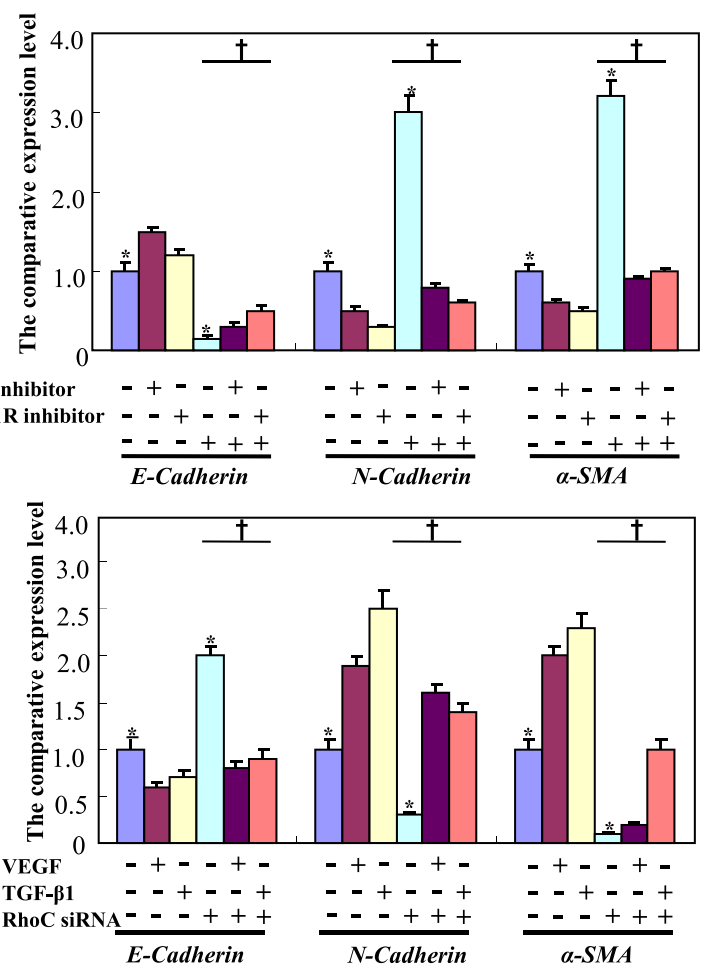

B
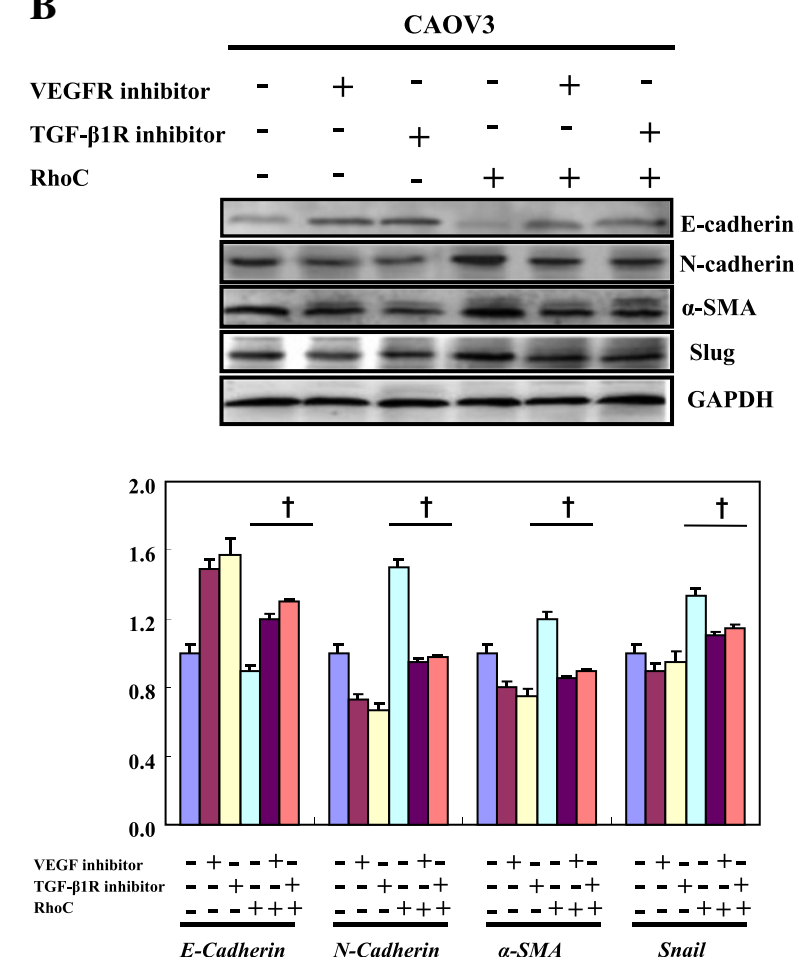
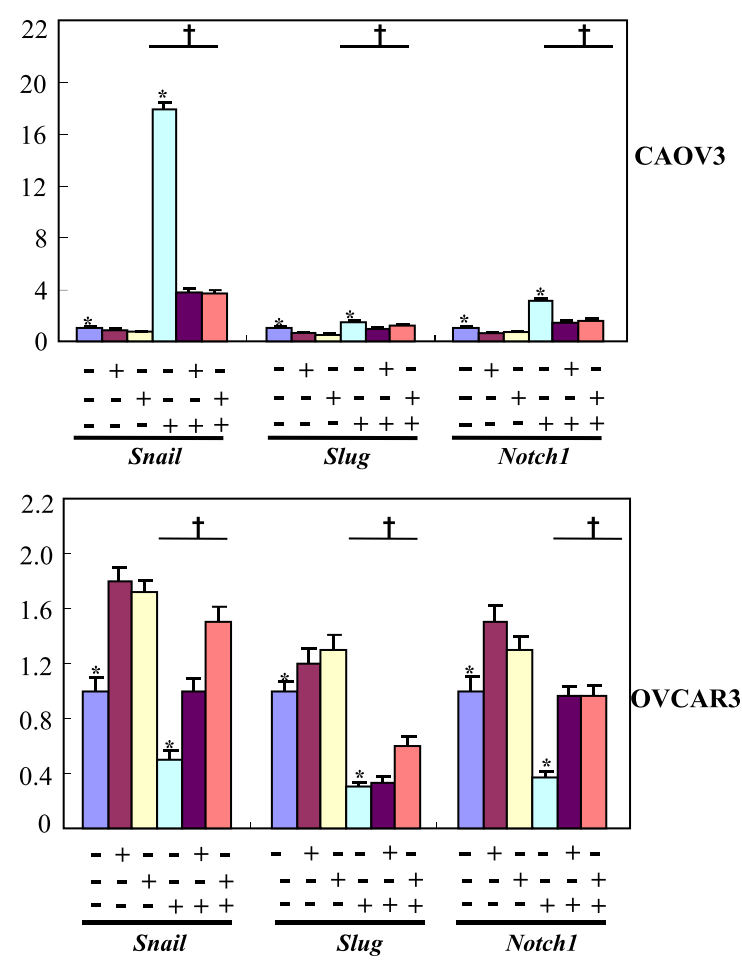

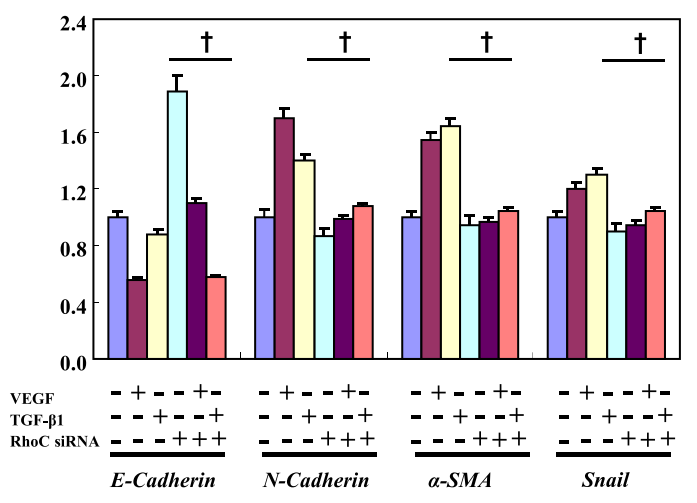

Figure 5 (See legend on next page.) 
(See figure on previous page.)

Figure 5 The RhoC-mediated roles of VEGF and TGF- $\beta 1$ in the expression of EMT-related molecules. In CAOV3 cells, VEGFR and TGF- $\beta 1 R$ inhibitors could up-regulate E-cadherin mRNA expression and down-regulated N-cadherin, a-SMA, snail and Notch1 mRNA expression, but their growth factors had the opposite effects in OVCAR3 cells by real- time PCR (A). According to Western blot and densitometric analysis, both inhibitors increased the E-cadherin expression, but decreased N-cadherin, a-SMA and Slug expression (B). Growth factors suppressed the E-cadherin expression, while enhanced the expression of N-cadherin, a-SMA and Slug (B). RhoC overexpression decreased the E-cadherin expression and increased the expression of N-cadherin, a-SMA, Slug and Notch1 in CAOV3 cells, while RhoC siRNA had the opposite effects in OVCAR3 cells (A and B). ${ }^{*}$ compared with treating groups, $p<0.05 ; \dagger$ compared with corresponding either RhoC- overexpressing or -hypoexpressing group.

light microscopy, while plasmid transfectants displayed a spindle appearance (Figure $1 \mathrm{E}, \mathrm{p}<0.05$ ). RhoC overexpression down-regulated E-cadherin mRNA expression and up-regulated $N$-cadherin and a-SMA mRNA expression in CAOV3 transfectants, compared with mock and control cells (Figure 1F). After RhoC siRNA treatment, E-cadherin mRNA expression was higher in OVCAR3 transfectants than control and mock cells by real-time PCR, while $N$-cadherin and $a-S M A$ mRNA expression was lower (Figure 1F).

\section{RhoC-mediated effects of VEGF and TGF- $\beta 1$ on EMT and related molecules in ovarian carcinoma cells}

TGF- $\beta 1 R$ or VEGFR inhibitors suppressed the proliferation of CAOV3 cells in both dose-dependent and timedependent manners, but TGF- $\beta 1$ or VEGF promoted proliferation of OVCAR3 cells and their transfectans (Figure 2). Exposure to both the receptor inhibitors increased the ratio of round CAOV3 cells and their transfectancts although both the growth factors caused elongation of OVCAR3 cells (Figure 3A). VEGFR or TGF- $\beta 1 \mathrm{R}$ inhibitors decreased the ability of CAOV3 cells and their RhoC transfectants to form lamellipodia (Figure 3B), migrate (Figure 4A, p < 0.05), and invade (Figure $4 \mathrm{~B}, \mathrm{p}<0.05$ ), while VEGF or TGF- $\beta 1$ enhanced lamellipodia formation (Figure $3 \mathrm{~B}, \mathrm{p}<0.05$ ), migration (Figure 4A) and invasion (Figure 4B, p<0.05) of OVCAR3 and their RhoC siRNA transfectants. Ectopic RhoC overexpression enhanced proliferation, migration, invasion and lamellipodia formation of CAOV3 cells regardless of the treatment of VEGFR or TGF- $\beta 1 \mathrm{R}$ inhibitor, whereas RhoC knockdown weakened above- mentioned biological events of OVCAR3 cells even with the exposure to VEGF or TGF- $\beta 1$ (Figures 2, 3, and 4).

In CAOV3 and its RhoC transfectant, VEGFR and TGF- $\beta 1 \mathrm{R}$ inhibitors up-regulated E-cadherin mRNA expression and down-regulated $N$-cadherin, $\alpha$-SMA, snail and Notch1 mRNA expression, but corresponding growth factors had the opposite effects in OVCAR3 and RhoC- knockdown transfectants based on real-time PCR (Figure 5A, p < 0.05). E-cadherin expression was increased and $\mathrm{N}$-cadherin, $\alpha$-SMA and Slug expression were decreased in CAOV3 and its transfectants treated by receptor inhibitors. Growth factors inhibited E-cadherin expression, while promoting $\mathrm{N}$-cadherin, $\alpha$-SMA and Slug expression (Figure 5B, p < 0.05). Immunofluorescence results for $\mathrm{E}$ - and $\mathrm{N}$-cadherin were similar to those shown by Western blot (Figure 6, p < 0.05). RhoC overexpression decreased the expression of the epithelial markers (E-cadherin) and increased mesenchymal markers (N-cadherin, $\alpha$-SMA, Slug and Notch1) in CAOV3 cells even exposed to VEGFR or TGF- $\beta 1 R$ inhibitor. In contrast, RhoC siRNA had the opposite effects in OVCAR3 cells, treated with or without VEGF or TGF- $\beta 1$ (Figures 5 and 6, $\mathrm{p}<0.05)$.

\section{Discussion and conclusions}

As reviewed, a possible role for $\mathrm{RhoC}$ was clarified in the EMT-related invasion and in metastasis because in vivo and vitro RhoC overexpression is associated with tumor cell invasion and metastasis [7]. In colon carcinoma, RhoC protein expression and subsequent activation were detected coincident with the loss of E-cadherin and acquisition of mesenchymal characteristics. A marked increase in $\mathrm{RhoC}$ expression was associated with the EMT of colon carcinoma cells and RhoC promoted post-EMT cell migration [14]. Here, we found the promoting effects of RhoC in EMT of ovarian carcinoma cells, evidenced by the alteration in morphological appearance and EMT markers (E-cadherin, N-cadherin and $\alpha$-SMA) in either RhoC-overexpressing or -hypoexpressing cells. In line with previous reports [16,17], forced RhoC overexpresion resulted in the faster migration, higher invasion and more lamellipodia formation for ovarian carcinoma cells, while RhoC knockdown did the opposite. In particular, our previous study demonstrated that the treatment with either RhoC siRNA or Rho inhibitor, Lovastatin reduced the mobility of ovarian carcinoma cell, OVCAR3, possibly through the down-regulation of MMP9 and VEGF $[9,10]$. These data suggested that RhoC might be a signaling protein in the EMT pathway of ovarian carcinoma cells.

Various reports showed that TGF- $\beta 1$ and VEGF might initiate the EMT of carcinoma cells [18-20]. In the present study, it was found that both TGF- $\beta 1 \mathrm{R}$ and VEGFR inhibitors decreased the aggressive phenotypes 


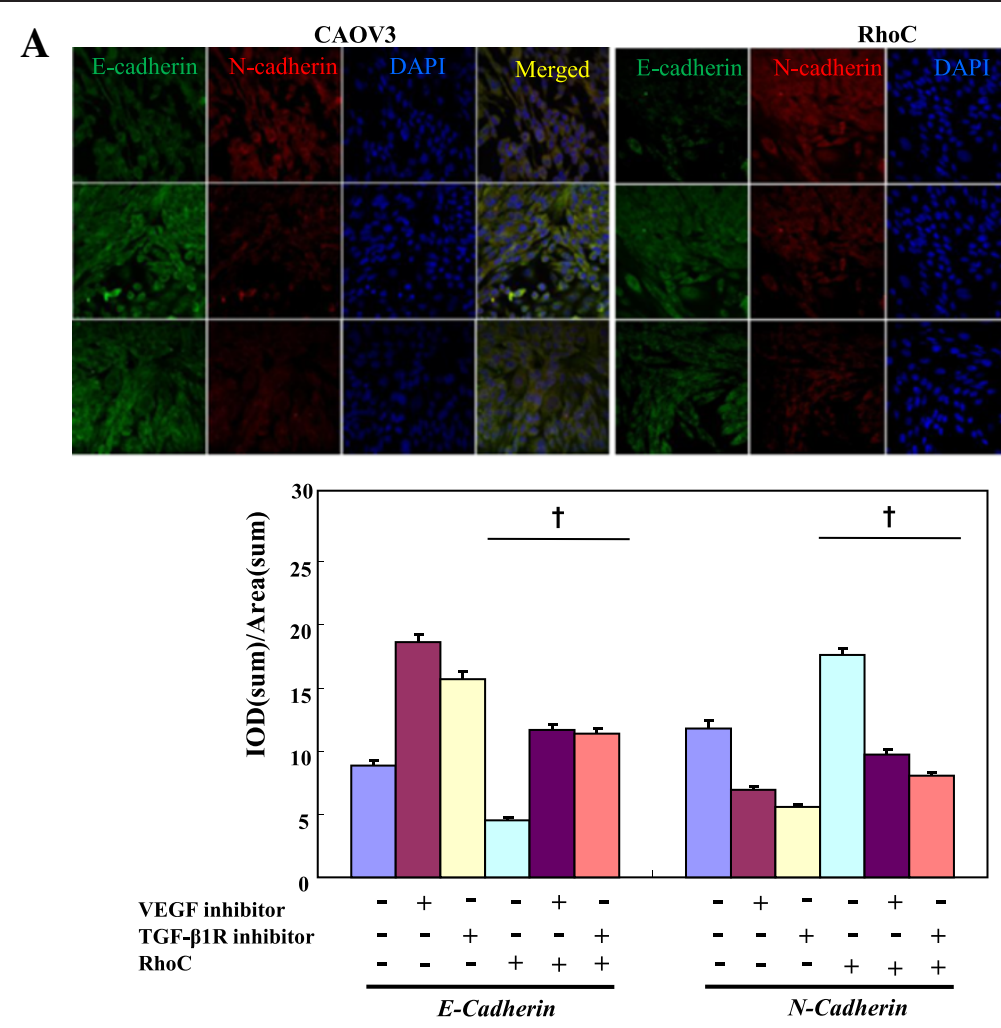

B

OVCAR3
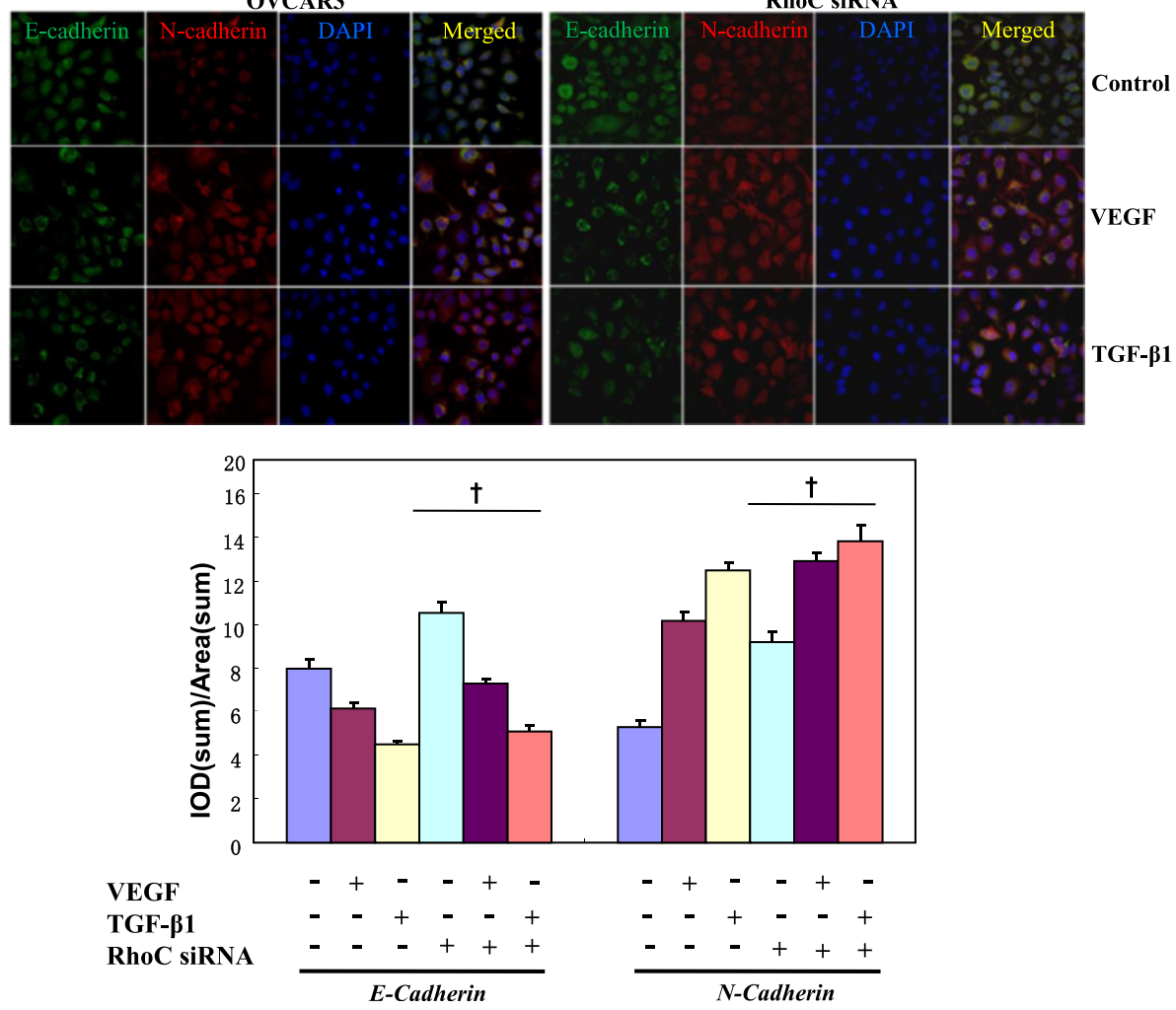

Figure 6 (See legend on next page.) 
(See figure on previous page.)

Figure 6 The RhoC-mediated roles of VEGF and TGF- $\beta 1$ in the expression of E-cadherin and N-cadherin by immunofluorescence. In CAOV3 cells, VEGFR and TGF- $\beta 1 R$ inhibitors could up-regulate the E-cadherin expression and down-regulated N-cadherin expression (A), but their growth factors had the opposite effects in OVCAR3 cells (B). RhoC overexpression decreased the E-cadherin expression and increased the expression of $\mathrm{N}$-cadherin in CAOV3 cells, while RhoC siRNA had the opposite effects in OVCAR3 cells (A and B). * compared with treating groups, $\mathrm{p}<0.05$; † compared with corresponding either RhoC- overexpressing or -hypoexpressing group.

(e.g. proliferation, migration, invasion and lamellipodia formation) in CAOV3 and its RhoC transfectants. In contrast, both TGF- $\beta 1$ and VEGF had the converse biological effects in OVCAR3 and RhoC-knockdown transfectants. Interestingly, RhoC siRNA might inhibit migration, invasion and lamellipodia formation of OVCAR3 treated with or without TGF- $\beta 1$ or VEGF, while RhoC overexpression might promote these events of CAOV3 cells even with the exposure to TGF- $\beta 1 R$ or VEGFR inhibitor. Mukai et al. [21] demonstrated that RhoC overexpression plays a critical role in the migration of hepatoma cells in rat ascites after the treatment of TGF- $\beta 1$. Wang et al. [22] showed that RhoC is the downstream regulator of VEGF in endothelial cells and is essential for angiogenesis induced by VEGF. These indicated that VEGF and TGF- $\beta 1$ might promote the migration, invasion and EMT of ovarian carcinoma cells, which is possibly regulated by RhoC.

To explore the molecular mechanisms about the role of VEGF and TGF- $\beta 1$ in EMT of ovarian carcinoma cells, we examined the EMT-related molecules in combination with quantitative PCR, Western blot and immunofluorescence. Consequently, it was found that both recombinant VEGF and TGF- $\beta 1$ could down-regulate E-cadherin expression, but up-regulate $N$-cadherin and $\alpha$-SMA expression with the opposite role of both their receptor inhibitors, supporting the regulatory effects of VEGF and TGF- $\beta$ on EMT of ovarian carcinoma cells. During EMT, the exposure to TGF- $\beta 1$ might up-regulate Snail and Slug expression and increase cell invasion [23]. The canonical TGF ß-Smad signaling might also regulate Snail and Slug expression [24]. Here, the exposure to VEGF or TGF- $\beta 1$ increased snail expression at both mRNA and protein levels, indicating RhoC also promote the event of EMT as a signal molecule. According to the literature, the activation of Notch-1 signaling contributes to the acquisition of EMT phenotype of pancreatic carcinoma cells [25]. Another study has provided evidences for the opinion that $\mathrm{RhoC}$ is an effector of Notch1 in cervical carcinoma cells [12]. Here, it was worth noting that VEGF and TGF- $\beta 1$ also enhanced Notch1 expression via $\mathrm{RhoC}$ protein, which will form a positive feedback loop for the initiation of EMT. After RhoCexpressing plasmid transfection, there appeared the downregulated expression of the epithelial markers and the up-regulated expression of mesenchymal markers in CAOV3 cells regardless of the exposure to VEGFR or
TGF- $\beta 1 \mathrm{R}$ inhibitor. In contrast, RhoC siRNA caused the opposite effects in OVCAR3 cells, even treated with both VEGF and TGF- $\beta 1$. Taken together, VEGF and TGF- $\beta 1$ were suggested to play an important role in EMT of ovarian carcinoma cells possibly via RhoC and final effectors, including snail and slug.

In summary, our study indicated that aberrant RhoC expression might be involved in EMT of ovarian cancer cells, initiated by TGF- $\beta 1$ and VEGF. The abovementioned three molecules should be considered as good targets to reverse EMT of ovarian carcinoma cell, which is useful and helpful for the treatment of the metastasis and recurrence of ovarian carcinoma.

\section{Competing interests}

The authors have declared that no competing interests exist.

\section{Authors' contributions}

HCZ designed the study and wrote the manuscript. WFG, YZ, HL, XFY, YLX, SZ, JML, ZTZ and HZS finished the experiments of cell culture, molecular and morphological examination, and animal model. FX, YPL and YT helped us with statistical analysis, English checking and manuscript correction. All authors read and approved the final manuscript.

\section{Acknowledgements}

This study was supported by Shenyang Science and Technology Grant (F11-264-1-10; F12-277-1-01); Liaoning Science and Technology Grant (2009225008-11); Natural Scientific Foundation of China (81172371; 81202049); and Grant-in aid for Scientific Research from the Ministry of Education, Culture, Sports and Technology of Japan (23659958).

\section{Author details}

${ }^{1}$ Cancer Research Center, The First Affiliated Hospital of Liaoning Medical University, 121001 Jinzhou, China. ${ }^{2}$ Key Laboratory of Brain and Spinal Cord Injury of Liaoning Province, The First Affiliated Hospital of Liaoning Medical University, 121001 Jinzhou, China. ${ }^{3}$ Department of Gynecology, The First Affiliated Hospital of China Medical University, 110001 Shenyang, China. ${ }^{4}$ Department of Oncological Medicine, The First Affiliated Hospital of China Medical University, 110001 Shenyang, China. ${ }^{5}$ Department of Physiology, School of Life Science and Biopharmaceutics, Shenyang Pharmaceutical University, 110016 Shenyang, China. ${ }^{6}$ Clinical Research Institute, Kanagawa Cancer Center, 241-0815 Yokohama, Japan.

Received: 2 December 2013 Accepted: 19 June 2014 Published: 1 July 2014

\section{References}

1. Menon U, Gentry-Maharaj A, Jacobs I: Ovarian cancer screening and mortality. JAMA 2011, 306(14):1544.

2. Bandera CA: Advances in the understanding of risk factors for ovarian cancer. J Reprod Med 2005, 50(5):399-406.

3. Huang RY, Chung VY, Thiery JP: Targeting pathways contributing to epithelial- mesenchymal transition (EMT) in epithelial ovarian cancer. Curr Drug Targets 2012, 13(13):1649-1653.

4. Savagner P: The epithelial-mesenchymal transition (EMT) phenomenon. Ann Oncol 2010, 21(Suppl 7):vii89-vii92. 
5. Wang Z, Li Y, Kong D, Sarkar FH: The role of Notch signaling pathway in epithelial- mesenchymal transition (EMT) during development and tumor aggressiveness. Curr Drug Targets 2010, 11(6):745-751.

6. Ouyang G, Wang Z, Fang X, Liu J, Yang CJ: Molecular signaling of the epithelial to mesenchymal transition in generating and maintaining cancer stem cells. Cell Mol Life Sci 2010, 67(15):2605-2618.

7. Bendris N, Arsic N, Lemmers B, Blanchard JM: Cyclin A2: Rho GTPases and EMT. Small GTPases 2012, 3(4):225-228.

8. Ridley AJ: Rho GTPases and actin dynamics in membrane protrusions and vesicle trafficking. Trends Cell Biol 2006, 16(10):522-529.

9. Zhao $\mathrm{Y}$, Zong ZH, Xu HM: RhoC expression level is correlated with the clinicopathological characteristics of ovarian cancer and the expression levels of ROCK-I, VEGF, and MMP9. Gynecol Oncol 2010, 116(3):563-571.

10. Zhao Y, Zheng HC, Chen S, Gou WF, Xiao L, Niu ZF: The role of RhoC in ovarian epithelial carcinoma: a marker for the carcinogenesis, progression, prognosis, target therapy. Gynecol Oncol 2013, 130(3):570-578.

11. Hakem A, Sanchez-Sweatman O, You-Ten A, Duncan G, Wakeham A, Khokha R, Mak TW: RhoC is dispensable for embryogenesis and tumor initiation but essential for metastasis. Genes Dev 2005, 19(17):1974-1979.

12. Srivastava S, Ramdass B, Nagarajan S, Rehman M, Mukherjee G, Krishna S: Notch1 regulates the functional contribution of RhoC to cervical carcinoma progression. Br J Cancer 2010, 102(1):196-205

13. Sequeira $L$, Dubyk CW, Riesenberger TA, Cooper CR, van Golen KL: Rho GTPases in PC-3 prostate cancer cell morphology, invasion and tumor cell diapedesis. Clin Exp Metastasis 2008, 25(5):569-579.

14. Bellovin DI, Simpson KJ, Danilov T, Maynard E, Rimm DL, Oettgen P, Mercurio AM: Reciprocal regulation of RhoA and RhoC characterizes the EMT and identifies RhoC as a prognostic marker of colon carcinoma. Oncogene 2006, 25(52):6959-6967.

15. Li W, Murai Y, Okada E, Matsui K, Hayashi S, Horie M, Takano Y: Modified and simplified western blotting protocol: use of intermittent microwave irradiation (IMWI) and 5\% skim milk to improve binding specificit y. Pathol Int 2002, 52(3):234-238.

16. Liu N, Zhang G, Bi F, Pan Y, Xue Y, Shi Y, Yao L, Zhao L, Zheng Y, Fan D: RhoC is essential for the metastasis of gastric cancer. J Mol Med (Berl) 2007, 85(10):1149-1156.

17. Islam M, Lin G, Brenner JC, Pan Q, Merajver SD, Hou Y, Kumar P, Teknos TN RhoC expression and head and neck cancer metastasis. Mol Cancer Res 2009, 7(11):1771-1780.

18. Singh A, Settleman J: EMT, cancer stem cells and drug resistance: an emerging axis of evil in the war on cancer. Oncogene 2010, 29(34):4741-475

19. Bates RC, Mercurio AM: The epithelial-mesenchymal transition (EMT) and colorectal cancer progression. Cancer Biol Ther 2005, 4(4):365-370.

20. Martin FT, Dwyer RM, Kelly J, Khan S, Murphy JM, Curran C, Miller N, Hennessy E, Dockery P, Barry FP, O'Brien T, Kerin MJ: Potential role of mesenchymal stem cells (MSCs) in the breast tumour microenvironment: stimulation of epithelial to mesenchymal transition (EMT). Breast Cancer Res Treat 2010, 124(2):317-326.

21. Mukai M, Endo H, Iwasaki T, Tatsuta M, Togawa A, Nakamura H, Inoue M: RhoC is essential for TGF-beta1-induced invasive capacity of rat ascites hepatoma cells. Biochem Biophys Res Commun 2006, 346(1):74-82.

22. Wang $W, W u F$, Fang $F$, Tao $Y$, Yang $L:$ RhoC is essential for angiogenesis induced by hepatocellular carcinoma cells via regulation of endothelial cell organization. Cancer Sci 2008, 99(10):2012-2018.

23. Naber HP, Drabsch Y, Snaar-Jagalska BE, ten Dijke P, van Laar T: Snail and Slug, key regulators of TGF- $\beta$-induced EMT, are sufficient for the induction of single-cell invasion. Biochem Biophys Res Commun 2013, 435(1):58-63.

24. Brandl M, Seidler B, Haller F, Adamski J, Schmid RM, Saur D, Schneider G: IKK(a) controls canonical TGF(B)-SMAD signaling to regulate genes expressing SNAIL and SLUG during EMT in panc1 cells. J Cell Sci 2010, 123(Pt 24):4231-4239.

25. Bao B, Wang Z, Ali S, Kong D, Li Y, Ahmad A, Banerjee S, Azmi AS, Miele L, Sarkar FH: Notch-1 induces epithelial-mesenchymal transition consistent with cancer stem cell phenotype in pancreatic cancer cells. Cancer Lett 2011, 307(1):26-36.

doi:10.1186/1471-2407-14-477

Cite this article as: Gou et al:: The role of RhoC in epithelial-tomesenchymal transition of ovarian carcinoma cells. BMC Cancer 2014 14:477.

\section{Submit your next manuscript to BioMed Central and take full advantage of:}

- Convenient online submission

- Thorough peer review

- No space constraints or color figure charges

- Immediate publication on acceptance

- Inclusion in PubMed, CAS, Scopus and Google Scholar

- Research which is freely available for redistribution 